\title{
THE TAIWAN-AMERICAN OCCULTATION SURVEY PROJECT STELLAR VARIABILITY. I. DETECTION OF LOW-AMPLITUDE $\delta$ SCUTI STARS
}

\author{
D.-W. Kim ${ }^{1,2,3}$, P. Protopapas ${ }^{1,2}$, C. Alcock ${ }^{1}$, Y.-I. Byun ${ }^{3}$, J. Kyeong ${ }^{4}$, B.-C. LeE ${ }^{4}$, N. J. Wright ${ }^{1}$, T. Axelrod ${ }^{5}$, \\ F. B. BiAnCo ${ }^{1,6}$, W.-P. Chen ${ }^{7}$, N. K. Coehlo ${ }^{8}$, K. H. CoOK ${ }^{9}$, R. DAvE ${ }^{2}$, S.-K. King ${ }^{10}$, T. LeE ${ }^{10}$, M. J. LeHNER ${ }^{1,6,10}$, H.-C. LiN ${ }^{7}$, \\ S. L. Marshall ${ }^{9,11}$, R. Porrata ${ }^{12}$, J. A. Rice ${ }^{8}$, M. E. Schwamb ${ }^{13}$, J.-H. WAng ${ }^{7,10}$, S.-Y. WAnG ${ }^{10}$, C.- Y. Wen $^{10}$, \\ AND Z.-W. ZHANG ${ }^{7,10}$ \\ ${ }^{1}$ Harvard Smithsonian Center for Astrophysics, Cambridge, MA 02138, USA \\ ${ }^{2}$ Initiative in Innovative Computing, School of Engineering and Applied Sciences, Harvard, Cambridge, MA 02138, USA \\ ${ }^{3}$ Department of Astronomy, Yonsei University, Seoul 120-749, Republic of Korea \\ ${ }^{4}$ Korea Astronomy \& Space Science Institute, Daejeon 305-348, Republic of Korea \\ ${ }^{5}$ Steward Observatory, 933 North Cherry Avenue, Room N204 Tucson, AZ 85721, USA \\ ${ }^{6}$ Department of Physics and Astronomy, University of Pennsylvania, 209 South 33rd Street, Philadelphia, PA 19104, USA \\ ${ }^{7}$ Institute of Astronomy, National Central University, No. 300, Jhongda Rd, Jhongli City, Taoyuan County 320, Taiwan \\ ${ }^{8}$ Department of Statistics, University of California Berkeley, 367 Evans Hall, Berkeley, CA 94720, USA \\ ${ }^{9}$ Institute for Geophysics and Planetary Physics, Lawrence Livermore National Laboratory, Livermore, CA 94550, USA \\ ${ }^{10}$ Institute of Astronomy and Astrophysics, Academia Sinica, P.O. Box 23-141, Taipei 106, Taiwan \\ ${ }^{11}$ Kavli Institute for Particle Astrophysics and Cosmology, 2575 Sand Hill Road, MS 29, Menlo Park, CA 94025, USA \\ ${ }^{12}$ Department of Astronomy, University of California Berkeley, 601 Campbell Hall, Berkeley, CA 94720, USA \\ ${ }^{13}$ Division of Geological and Planetary Sciences, California Institute of Technology, 1201 E. California Blvd., Pasadena, CA 91125, USA \\ Received 2009 September 29; accepted 2009 December 6; published 2010 January 14
}

\begin{abstract}
We analyzed data accumulated during 2005 and 2006 by the Taiwan-American Occultation Survey (TAOS) in order to detect short-period variable stars (periods of $\lesssim 1 \mathrm{hr}$ ) such as $\delta$ Scuti. TAOS is designed for the detection of stellar occultation by small-size Kuiper Belt Objects and is operating four $50 \mathrm{~cm}$ telescopes at an effective cadence of $5 \mathrm{~Hz}$. The four telescopes simultaneously monitor the same patch of the sky in order to reduce false positives. To detect short-period variables, we used the fast Fourier transform algorithm (FFT) in as much as the data points in TAOS light curves are evenly spaced. Using FFT, we found 41 short-period variables with amplitudes smaller than a few hundredths of a magnitude and periods of about an hour, which suggest that they are low-amplitude $\delta$ Scuti stars. The light curves of TAOS $\delta$ Scuti stars are accessible online at the Time Series Center Web site (http://timemachine.iic.harvard.edu).
\end{abstract}

Key words: stars: variables: delta Scuti - methods: data analysis - surveys

\section{INTRODUCTION}

$\delta$ Scuti stars (hereinafter, $\delta$ Sct stars) are pulsating variables inside the classical instability strip and on or close to the main sequence. They are typically placed lower on the instability strip than RR Lyrae stars or Cepheids and thus they are fainter than RR Lyrae stars or Cepheids. Their spectral types are between $\mathrm{A}$ and late $\mathrm{F}$. Their periods are between $\sim 0.02$ days and $\sim 0.25$ days, which is relatively shorter than other types of variables (e.g., $\gamma$ Dor; Henry et al. 2001). Based on these characteristics, $\delta$ Sct stars can be separated from other types of variable stars such as RR Lyrae, $\beta$ Cepheid, $\gamma$ Dor, etc. (Breger 2000a).

The majority of the $\delta$ Sct stars are low-amplitude $\delta$ Sct stars (LADS) with amplitudes from a millimagnitude to a few tens of a millimagnitude. LADS are mainly non-radial $p$-mode pulsators (Breger 2000a). Another subgroup of $\delta$ Sct stars is the highamplitude $\delta$ Sct stars (HADS), whose amplitudes are bigger than $\sim 0.3$ mag. HADS are radial pulsators (Breger 2000a; Rodríguez et al. 1996). In addition to LADS and HADS, there is another interesting type of pulsation star called SX Phe variable stars, which exhibits a type of pulsation similar to the $\delta$ Sct stars. They are relatively old and evolved Population II stars, whereas most of the $\delta$ Sct stars are Population I stars. Stellar evolutionary theory is not yet successful at explaining these SX Phe variable stars (Rodríguez \& López-González 2000). Most of the SX Phe show similar properties with HADS such as high amplitude and short period. More detailed review of $\delta$ Sct stars is presented in Breger (2000a, and references therein).

Because of their great number of radial and non-radial modes, it is known that $\delta$ Sct stars are suitable for asteroseismology research, which enables study of stellar interior structures (Brown \& Gilliland 1994). For a better understanding of pulsating $\delta$ Sct stars and thus stellar structure, several authors studied $\delta$ Sct stars and detected their multiple frequencies of pulsation using either ground-based observations or space-based observations (Breger et al. 2002, 2005; Ripepi et al. 2003; Buzasi et al. 2005; Bruntt et al. 2007; Pribulla et al. 2008). Due to the better photometric precision, space-based observation data show better results on the analysis of multiple frequencies than ground-based observation data (Bruntt et al. 2007; Pribulla et al. 2008). However, some authors have pointed out that ground-based observations using multiple-site telescopes are still valuable because, with a baseline longer than space-based observations, they are useful for detecting long-period pulsation (for more details, see Breger et al. 2005; Bruntt et al. 2007, and references therein). Moreover, by parameterizing the amplitude ratio and the phase differences in different filters (e.g., $u b v y$ ), it is possible to derive the spherical harmonic degree, $l$ (Garrido et al. 1990; Balona \& Evers 1999; Moya et al. 2004), which is an important parameter for the asteroseismology studies. Therefore, ground-based telescopes that are more feasible for multiple-site and multiple-filter observations (e.g., Delta Scuti Network, Zima et al. 2002) are nonetheless useful for the 
identification of pulsation modes and thus for the study of interior structures.

Another interesting feature of $\delta$ Sct stars is that some of them show period and amplitude variations (Breger \& Pamyatnykh 1998; Breger 2000b; Arentoft et al. 2001). The period variation $(1 / P) d P / d t$, based on observations, is about $10^{-7}$ per year for both period increases and decreases with equal distribution. On the other hand, theoretical models give 10 times smaller period variation than observed; they also predict that period increases should be dominant over period decreases (Breger \& Pamyatnykh 1998). Amplitude variations and timescales of the variations are different from star to star, ranging from a few millimagnitudes to several tens of millimagnitudes and from a few tens of days to a few hundreds of days (Arentoft et al. 2001). These period and amplitude variations are thought to be caused not by evolutionary effects but by some other mechanism (e.g., light-time effect because of the orbital motion in binaries or nonlinear mode interactions). However, the true origin of the variations is still unknown. For more details, see Breger \& Pamyatnykh (1998) and references therein.

McNamara et al. (2007) investigated HADS in the Large Magellanic Cloud (LMC) and their period-luminosity $(P-L)$ relation to test if they can be used as the standard distance candles. They found that the distance modulus for LMC derived using $\delta$ Sct stars is consistent with the distance moduli for LMC derived using RR Lyrae and Cepheids, which implies the $P-L$ relation of $\delta$ Sct stars can help to determine distances of longdistance objects such as objects in the LMC.

In this paper, we present the detection of $41 \delta$ Sct candidate stars from the Taiwan-American Occultation Survey (TAOS) data accumulated during 2005 and 2006 observation (hereinafter, TAOS $\delta$ Sct stars). Among the 41 detections, there is one previously known "suspected variable" star, NSV 3816, from the Suspected Variable Stars and Supplement (Samus et al. 2009, no period or type is provided in that catalog). The rest of the 40 TAOS $\delta$ Sct stars are newly detected by this study. Only 14 of the detected TAOS $\delta$ Sct stars have spectral types. Twelve of those have spectral types from A to $\mathrm{F}$, which are typical for $\delta \mathrm{Sct}$ stars. The remaining two have B8 and G5 spectral types, which are peculiar spectral types. Using spectroscopic instrumentsBOES (Kim et al. 2007) and FAST (Fabricant et al. 1998) —we obtained spectra for those two stars. As a result we found that the B8 star is an A5 star and the G5 star is an F0 star. Even though the rest of the detected stars do not have spectral-type information, their low amplitudes, short periods, and morphologies of light curves strongly suggest that they are LADS.

In Section 2, we present a TAOS overview, data reduction processes, and the detection algorithm we used to detect $\delta$ Sct stars in TAOS two-year data. We provide a list of the detected TAOS $\delta$ Sct stars and their physical parameters (e.g., magnitude, period, amplitude, spectral type, etc.) in Section 3. In Section 4, we present summaries.

\section{TAOS $\delta$ Sct STARS}

\subsection{TAOS Overview}

TAOS aims to detect stellar occultations caused by smallsized Kuiper Belt Objects (KBOs) at a distance of Neptune's orbit or beyond (Alcock et al. 2003; Chen et al. 2007; Lehner et al. 2009). Because of the short duration $(<1 \mathrm{~s})$ and the rareness of occultation events, TAOS monitors several hundreds of stars in a wide field of view $\left(3 \mathrm{deg}^{2}\right)$ with a high sampling rate. To reduce false positives, TAOS uses four $50 \mathrm{~cm}$ telescopes which simultaneously monitor the same patch of the sky. Due to the high sampling rate, TAOS data are also useful for detecting short-period variable stars such as $\delta$ Sct stars. Moreover, TAOS telescopes keep monitoring the same field up to $1.5 \mathrm{hr}$ and can thus obtain full-phase light curves of variable stars whose periods are shorter than $1.5 \mathrm{hr}$. To detect such short-period variable stars, we analyzed TAOS data accumulated during 2005 and 2006. The data set consists of 117 TAOS observation fields, which cover $351 \mathrm{deg}^{2}$ of the sky. It consists of $\sim 200$ runs, where a run is a set of multiple (two or three ${ }^{14}$ ) telescope observations for a given field and a given date. Note that the TAOS telescopes occasionally visit the same observation field multiple times according to the telescopes' observation schedules, which enables detecting the same variable stars multiple times. In such a case, we are able to derive multiple frequencies of the stars as explained in Section 3.1.

\subsection{Data Reduction}

To detect periodic signals, we analyzed the light curves generated by the TAOS photometry pipeline (Zhang et al. 2009). The pipeline was developed by the collaboration to extract light curves of each star from zipper images. The zipper images are generated by the unique telescope operation mode called zipper mode which was developed in order to achieve highspeed photometry (Lehner et al. 2009).

After obtaining the light curves using the TAOS photometry pipeline, we applied further cuts to the light curves. Some of the individual measurements are flagged as invalid. This happens when the star moves out of the field of view because of temporary telescope vibrations or tracking error, thus yielding no photometrical measurements. We therefore applied a B-spline (de Boor 1978) and replaced the flagged measurements with values interpolated from the spline fit. After the interpolation process, in order to increase the signal-to-noise ratio $(\mathrm{S} / \mathrm{N})$, we binned each light curve using a fifty-point window (10 s). During the binning process, we used the average time of the 50 data points as the time of the binned data.

We then removed the systematic variations that are common across light curves of the same run. Such systematic variations, which we call trends, could be caused by air mass, temporary telescope vibrations, noise in CCD images, etc. To remove such trends, we applied the Photometric DeTrending algorithm (PDT, Kim et al. 2009) to each individual run. PDT first calculates the correlation between whole light curves as a measure of similarity between light curves. PDT then uses the hierarchical clustering algorithm (Jain et al. 1999) to group similar light curves together and determines one master trend per group by summing weighted light curves in the group. Using the determined master trends, PDT finally removes trends from each individual light curve by minimizing the residual between the master trends and the light curve. For more details about PDT, see Kim et al. (2009).

Figure 1 shows an example of a TAOS $\delta$ Sct star's light curve before and after the detrending process. The $x$-axis is time in minutes, and the $y$-axis is flux. As the figure shows, periodic signals are clearly recovered after detrending. We show the errors for each photometric measurement before detrending, propagated from the errors estimated by the TAOS photometry pipeline (Zhang et al. 2009).

\footnotetext{
${ }^{14}$ During 2005 and 2006 observational season, one of the four TAOS telescopes was not operational.
} 

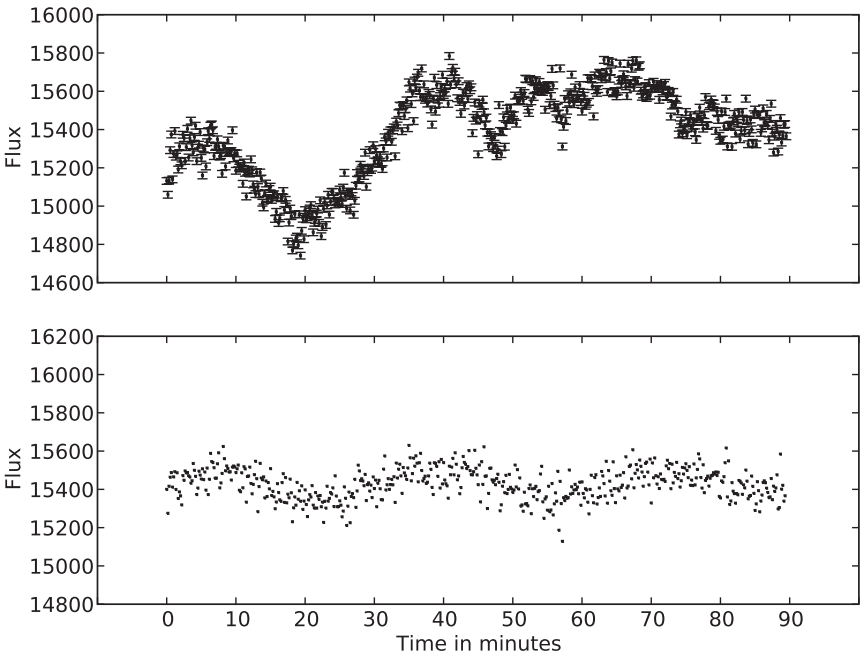

Figure 1. Example light curve of a TAOS $\delta$ Sct star. The $x$-axis is time in minutes and the $y$-axis is flux. The top panel is the light curve before detrending and the bottom panel is the light curve after detrending. The periodic signals contaminated by unstable weather (e.g., moving clouds) are successfully recovered after detrending. We show the errors for each photometric measurement of the raw light curve.

\subsection{Detection of Short-period Variable Stars}

After we finished the preprocessing, explained in the previous section, we applied the fast Fourier transform algorithm (FFT; Brigham 1974) to each light curve in order to detect periodic signals. Note that the individual measurements of TAOS light curves are evenly spaced with a $5 \mathrm{~Hz}$ sampling rate. ${ }^{15}$ Thus FFT is appropriate for the detection of periodic signals. We focused on the detection of short-period variable stars whose periods are $\lesssim 1.5 \mathrm{hr}$ because TAOS monitors a given field for a maximum of $1.5 \mathrm{hr}$.

We describe the basic steps of the detection process below.

1. We apply FFT to each detrended light curve and derive the power spectrum of the light curve. We then examine if there exists a frequency (or frequencies) whose power is bigger than five times the standard deviation of powers of the background frequencies. The standard deviation of powers is calculated after removing outliers using $3 \sigma$ clipping. ${ }^{16}$ We identify the star as a variable candidate if there is a frequency higher than five times the standard deviation.

2. For each candidate variable, we check if the periodic signal is detected on the other telescopes' light curves of the same run. If it is not detected by the other telescopes, we remove the star from the candidate list.

3 . We visually inspect all raw zipper images for the candidates and remove false positives caused by moving asteroids, photometry defects, or other contamination due to various noise sources. For instance, the flux of stars in the neighborhood of fast-moving objects could be increased and decreased within an hour, which resembles periodic signals.

4. We cross-match all of the candidates with SIMBAD (Wenger et al. 2000) and remove the false positives that are confirmed to be other types of variable stars (e.g., eclipsing binary stars).

\footnotetext{
15 Binned light curves are evenly spaced as well.

16 Those outliers are only removed for the calculation of the standard deviation. They are included in the search of periodic signals.
}

5. Finally, we remove the variable stars whose periods are longer than $1.5 \mathrm{hr}$.

\section{DETECTION RESULTS}

With the detection algorithm described in the previous section, we found $41 \delta$ Sct candidate stars whose periods are shorter than $1.5 \mathrm{hr}$ and whose amplitudes are within a few hundredth of a magnitude (hereinafter, TAOS $\delta$ Sct stars). Among those 41 TAOS $\delta$ Sct stars, one of them is a previously suspected variable star, NSV 3816, from the Suspected Variable stars and Supplement (Samus et al. 2009). However, the period and amplitude of NSV 3816 have never been published before. The remaining 40 TAOS $\delta$ Sct stars are newly detected by this study.

After the TAOS $\delta$ Sct stars were identified, we extracted the physical parameters of each star by cross-matching them with various astronomical catalogs. We show catalogs we used in Table 1 . We found that 12 of the detected 41 TAOS $\delta$ Sct stars have spectral types from A0 to F5, which are typical spectral types for $\delta$ Sct stars. Unfortunately, the rest of them, except for two peculiar $\delta$ Sct stars-discussed in Section 3.3-do not have spectral information. Nevertheless, their short period and low amplitude strongly suggest that they are LADS rather than other types of variables, such as RR Lyrae or Cepheids, whose periods and amplitudes are relatively longer and larger than those of $\delta$ Sct stars.

As a byproduct of our analysis, we detected a previously known variable star with $\delta$ Sct pulsation, GM Leo, which is actually a $\lambda$ Bootis star (Handler et al. 2000). Some $\lambda$ Bootis stars show $\delta$ Sct pulsations (Paunzen 2004) and can have spectral types from late B to early $\mathrm{F}$, which makes it difficult to distinguish them from $\delta$ Sct stars. In such cases, there are no clear differences between $\delta$ Sct stars and $\lambda$ Bootis stars except the metal abundance (Balona 2004); $\lambda$ Bootis stars show weak metal lines such as the Mg II $\lambda 4481$ line (Paunzen 2004).

We also checked several preexisting catalogs of $\delta$ Sct stars to see if there are previously known $\delta$ Sct stars in the TAOS observation fields. Table 2 shows the preexisting catalogs we checked. As a result of this search, we found only one previously known $\delta$ Sct pulsation star to be in the TAOS observation fields. That turns out to be GM Leo, which, as mentioned above, we successfully detected. Although GCVS classified GM Leo as $\delta$ Sct star based on the work by Handler et al. (2000), Handler et al. (2000) in their paper claimed GM Leo is not a $\delta$ Sct star but a $\lambda$ Bootis star. Thus we removed GM Leo from our detection list.

\subsection{List of the Detected $41 \delta$ Sct Stars}

Table 3 shows the 41 TAOS $\delta$ Sct stars' physical information such as positions, magnitudes, frequencies, amplitudes, spectral types, etc.

We used the FFT algorithm to detect periodic signals; however, we used PERIOD04 to derive their physical parameters such as period and amplitude. ${ }^{17}$ This is because PERIOD04 improves the frequency by fitting the light curve with a combination of sine curves. Moreover PERIOD04 also provides errors for the derived frequencies. Figure 2 shows a comparison result of power spectra derived from an FFT method and PERIOD04 for a single TAOS $\delta$ Sct star. The $x$-axis is frequency in counts/day and the $y$-axis is scaled power. The solid line is

\footnotetext{
17 Since PERIOD04 gives half of the full amplitudes, we doubled amplitudes
} derived by PERIOD04 as Rodríguez et al. (2000) and other authors do. 
Table 1

Catalogs Used to Extract Additional Parameters

\begin{tabular}{lc}
\hline \hline \multicolumn{1}{c}{ Catalog } & Reference \\
\hline GCVS & Perryman \& ESA (1997) \\
All-Sky Compiled Catalogue of 2.5 million stars & Kharchenko (2001) \\
HD & Cannon \& Pickering (1993) \\
Catalog of Stellar Spectral Classifications & Skiff (2009) \\
Tycho-2 Catalogue of the 2.5 Million Brightest Stars & Høg et al. (2000) \\
Guide Star Catalog (GSC) & Lasker et al. (2008) \\
USNO-B 1.0 & Monet et al. (2003) \\
SAO Star Catalog J2000 & SAO Staff (1995) \\
Catalog of Projected Rotational Velocities & Glebocki \& Stawikowski (2000) \\
Rotational Velocity Determinations for $118 \delta$ Sct Variables & Bush \& Hintz (2008)
\end{tabular}

Table 2

Preexisting Catalogs of $\delta$ Sct Stars

\begin{tabular}{|c|c|c|c|}
\hline Catalog & Source Surveys & Number of $\delta$ Sct Stars & Reference \\
\hline $\mathrm{R} 2000^{\mathrm{a}}$ & MACHO, OGLE, Hipparcos, etc & $\sim 600$ & (Rodríguez et al. 2000) \\
\hline ROTSE $^{b}$ & ROTSE & 6 & (Jin et al. 2003) \\
\hline $\mathrm{ASAS}^{\mathrm{c}}$ & ASAS & $\sim 500$ & (Pojmanski et al. 2006) \\
\hline $\mathrm{GCVS}^{\mathrm{d}}$ & Various surveys & $\sim 500$ & (Samus et al. 2009) \\
\hline TAOS & TAOS & 41 & This paper \\
\hline \multicolumn{4}{|l|}{ Others } \\
\hline & 5 new $\gamma$ Doradus and 5 new $\delta$ Sct survey & 5 & (Henry et al. 2001) \\
\hline & Case study for HD 173977 & 1 & (Chapellier et al. 2004) \\
\hline & Case study for HD 8801 & 1 & (Henry \& Fekel 2005) \\
\hline & The first HADS in an eclipsing binary star & 1 & (Christiansen et al. 2007) \\
\hline & Variable stars in NGC 2099 & 9 & (Kang et al. 2007) \\
\hline & Transit survey of M37 & 2 & (Hartman et al. 2008) \\
\hline & ASAS variable stars in the Kepler field of view & 4 & (Pigulski et al. 2009) \\
\hline
\end{tabular}

Notes.

a The catalog compiled by Rodríguez et al. (2000).

b Robotic Optical Transient Search Experiment.

c All-Sky Automated Survey.

${ }^{\mathrm{d}}$ General Catalog of the Variable Stars.

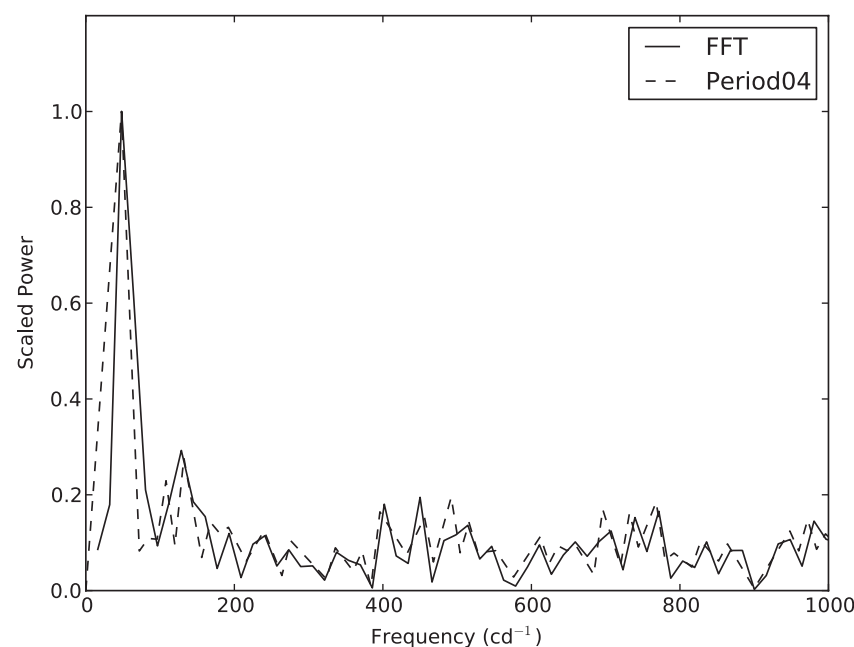

Figure 2. Comparison result of the power spectrum derived from an FFT method and from PERIOD04. The $x$-axis is frequency in counts/day and the $y$-axis is scaled power. The solid line is the power spectrum derived from an FFT method, and the dashed line is the power spectrum derived from PERIOD04. The two spectra appear almost identical.

a power spectrum derived from an FFT method, and the dashed line is a power spectrum derived from PERIOD04. As the figure shows, the two spectra are almost consistent.

As we mentioned in the previous section, TAOS operates multiple telescopes simultaneously monitoring the same patch of the sky. Thus we have a maximum of three simultaneous light curves for all TAOS $\delta$ Sct stars for a given zipper run. To derive more precise frequencies and amplitudes, for each identified $\delta$ Sct star we summed the light curves from each of the telescopes. Moreover, the TAOS telescopes occasionally visit same fields multiple times. In such cases, we merge all corresponding normalized light curves ${ }^{18}$ of each identified $\delta$ Sct star into a single but longer light curve. Having longer light curves we were able to extract multiple frequencies using PERIOD04. Among the extracted frequencies, we selected the frequencies whose $\mathrm{S} / \mathrm{N}$ is bigger than 5 . The $\mathrm{S} / \mathrm{N}$ of each frequency was calculated using PERIOD04 as well. ${ }^{19}$ As a result, we found 16 TAOS $\delta$ Sct stars having multiple frequencies (see Table 3). Note that we did not attempt to extract multiple frequencies if the star is detected only once (i.e., detected in only a single zipper run). It is also worth mentioning that we lose detectability on relatively long-period pulsations because we normalized the light curves while merging them.

All detected $\delta$ Sct stars are relatively bright as shown in the table (the faintest star's $m_{V}$ is around 12). This is because the limiting magnitude of TAOS zipper mode is relatively bright at $\sim 13.5$ (Lehner et al. 2009) and also because high $\mathrm{S} / \mathrm{N}$ is needed to detect low-amplitude variations of a few millimagnitudes. To

\footnotetext{
18 We normalized each light curve by their mean values.

19 Although other authors have suggested a threshold of $\mathrm{S} / \mathrm{N}>4$ (Breger et al. 1993; Christiansen et al. 2007), we empirically found that a threshold of $\mathrm{S} / \mathrm{N}>4$ produces false positives and thus we set the $\mathrm{S} / \mathrm{N}$ threshold to 5 .
} 
Table 3

TAOS $\delta$ Sct Stars in TAOS Two-year Data

\begin{tabular}{|c|c|c|c|c|c|c|c|c|c|c|c|c|}
\hline No. & $\mathrm{ID}^{\mathrm{a}}$ & $\begin{array}{c}\text { R.A. } \\
\text { (hh:mm:ss) }\end{array}$ & $\begin{array}{c}\text { Decl. } \\
\text { (dd:mm:ss) }\end{array}$ & $m_{V}$ & $m_{B}$ & $\begin{array}{c}\text { Frequency } \\
\left(\mathrm{cd}^{-1}\right)\end{array}$ & $\begin{array}{c}\Delta m_{V}^{\mathrm{b}} \\
(\mathrm{mmag})\end{array}$ & $\mathrm{S} / \mathrm{N}^{\mathrm{c}}$ & $\begin{array}{l}\text { Epoch } \\
\text { (MJD) }\end{array}$ & Spectral Type & \#/\#和 & Note \\
\hline 1 & 124.00003 & $00: 52: 40$ & $+06: 39: 55$ & 8.89 & 9.20 & $24.421 \pm 3.975 \mathrm{e}^{-4}$ & $3.83 \pm 0.20$ & 6.5 & 53626.7469 & A2 & $4 / 7$ & \\
\hline 2 & 038.00124 & 02:56:54 & $+34: 23: 20$ & 11.60 & 12.00 & $23.598 \pm 4.775 \mathrm{e}^{-1}$ & $7.95 \pm 0.44$ & 11.9 & 53678.6909 & & $1 / 2$ & \\
\hline 3 & 053.00009 & 03:37:02 & $+18: 21: 51$ & 8.48 & 8.80 & $20.544 \pm 2.314 \mathrm{e}^{-4}$ & $5.99 \pm 0.14$ & 18.5 & 53671.7396 & A2 & $3 / 8$ & \\
\hline \multirow[t]{4}{*}{4} & 059.00115 & 03:42:41 & $+17: 55: 01$ & 12.12 & 12.60 & $25.836 \pm 7.587 \mathrm{e}^{-4}$ & $9.19 \pm 0.68$ & 13.6 & 54021.6614 & & $3 / 8$ & \\
\hline & & & & & & $32.116 \pm 8.434 \mathrm{e}^{-4}$ & $7.97 \pm 0.66$ & 11.8 & 54021.6579 & & & \\
\hline & & & & & & $12.034 \pm 1.291 \mathrm{e}^{-3}$ & $4.94 \pm 0.58$ & 7.5 & 54021.5979 & & & \\
\hline & & & & & & $40.403 \pm 1.713 \mathrm{e}^{-3}$ & $3.53 \pm 0.62$ & 5.25 & 54021.6523 & & & \\
\hline \multirow[t]{2}{*}{5} & 059.00005 & 03:46:01 & $+18: 34: 00$ & 9.14 & 9.46 & $33.115 \pm 2.022 \mathrm{e}^{-4}$ & $8.00 \pm 0.20$ & 15.7 & 54006.7013 & A5 & $7 / 8$ & $\mathrm{~B} 8^{\mathrm{e}}$ \\
\hline & & & & & & $18.068 \pm 4.793 \mathrm{e}^{-4}$ & $3.26 \pm 0.20$ & 6.4 & 54006.7020 & & & \\
\hline \multirow[t]{3}{*}{6} & 049.00056 & 04:03:21 & $+19: 21: 31$ & 10.91 & 11.48 & $24.205 \pm 3.694 \mathrm{e}^{-4}$ & $9.15 \pm 0.34$ & 13.7 & 54029.8147 & & $6 / 16$ & \\
\hline & & & & & & $17.761 \pm 6.312 \mathrm{e}^{-4}$ & $4.98 \pm 0.36$ & 7.5 & 54029.7806 & & & \\
\hline & & & & & & $29.544 \pm 8.243 \mathrm{e}^{-4}$ & $4.00 \pm 0.34$ & 6.0 & 54029.8094 & & & \\
\hline \multirow[t]{2}{*}{7} & 068.00053 & 04:30:06 & $+20: 55: 00$ & 11.45 & 12.36 & $32.504 \pm 2.402 \mathrm{e}^{-5}$ & $10.17 \pm 0.28$ & 15.5 & 53643.8513 & F0 & $8 / 15$ & $G 5^{\mathrm{f}}$ \\
\hline & & & & & & $26.117 \pm 5.939 \mathrm{e}^{-5}$ & $4.10 \pm 0.28$ & 6.24 & 53643.8542 & & & \\
\hline 8 & 060.00151 & 04:48:37 & $+21: 10: 33$ & 11.44 & 11.64 & $43.180 \pm 3.709 \mathrm{e}^{-4}$ & $5.13 \pm 0.26$ & 8.4 & 54012.7340 & F5 & $6 / 32$ & \\
\hline \multirow[t]{3}{*}{9} & 022.00001 & $04: 56: 14$ & $+21: 34: 20$ & 7.34 & 7.70 & $21.582 \pm 1.709 \mathrm{e}^{-4}$ & $3.97 \pm 0.12$ & 12.9 & 54021.7647 & F0 & $7 / 25$ & \\
\hline & & & & & & $21.146 \pm 3.268 \mathrm{e}^{-4}$ & $2.15 \pm 0.12$ & 6.94 & 54021.7495 & & & \\
\hline & & & & & & $40.637 \pm 4.339 \mathrm{e}^{-4}$ & $1.57 \pm 0.12$ & 5.12 & 54021.7926 & & & \\
\hline 10 & 020.00206 & 05:08:38 & $+22: 49: 37$ & 11.85 & 12.16 & $53.575 \pm 5.075 \mathrm{e}^{-1}$ & $7.62 \pm 0.46$ & 9.9 & 53705.6765 & & $1 / 3$ & \\
\hline 11 & 020.00141 & 05:09:24 & $+23: 16: 05$ & 11.40 & 12.29 & $31.535 \pm 6.862 \mathrm{e}^{-1}$ & $6.87 \pm 0.52$ & 5.7 & 53705.6877 & & $1 / 3$ & \\
\hline \multirow[t]{2}{*}{12} & 021.00011 & 05:09:40 & $+21: 50: 05$ & 8.99 & 9.21 & $41.754 \pm 1.493 \mathrm{e}^{-4}$ & $8.05 \pm 0.18$ & 17.7 & 53975.7771 & & $5 / 7$ & \\
\hline & & & & & & $37.264 \pm 4.476 \mathrm{e}^{-4}$ & $2.73 \pm 0.18$ & 5.9 & 53975.7883 & & & \\
\hline 13 & 020.00135 & $05: 10: 14$ & $+23: 01: 24$ & 10.90 & 11.78 & $39.254 \pm 6.865 \mathrm{e}^{-1}$ & $5.11 \pm 0.40$ & 6.3 & 53705.6974 & & $1 / 3$ & \\
\hline 14 & 024.00234 & $05: 15: 19$ & $+22: 53: 41$ & 11.93 & 12.39 & $25.804 \pm 2.471 \mathrm{e}^{-1}$ & $35.35 \pm 1.04$ & 12.8 & 53679.7864 & & $1 / 2$ & \\
\hline 15 & 160.00106 & 06:01:30 & $+21: 27: 38$ & 10.38 & 10.69 & $21.063 \pm 3.878 \mathrm{e}^{-4}$ & $7.44 \pm 0.22$ & 13.9 & 53680.8125 & & $3 / 8$ & \\
\hline \multirow[t]{3}{*}{16} & 160.00004 & 06:04:05 & $+21: 29: 39$ & 7.81 & 7.97 & $25.827 \pm 5.607 \mathrm{e}^{-4}$ & $4.70 \pm 0.16$ & 15.5 & 53680.8193 & & $3 / 8$ & \\
\hline & & & & & & $22.395 \pm 7.432 \mathrm{e}^{-4}$ & $3.55 \pm 0.14$ & 11.7 & 53680.8406 & & & \\
\hline & & & & & & $38.868 \pm 1.077 \mathrm{e}^{-3}$ & $1.59 \pm 0.12$ & 5.2 & 53680.8298 & & & \\
\hline 17 & 160.00199 & $06: 04: 26$ & $+21: 21: 55$ & 10.96 & 11.16 & $43.741 \pm 9.070 \mathrm{e}^{-4}$ & $4.12 \pm 0.26$ & 11.0 & 53680.8119 & & $3 / 8$ & \\
\hline 18 & 052.00132 & 07:39:07 & $+21: 39: 20$ & 11.14 & 11.67 & $23.802 \pm 3.343 \mathrm{e}^{-3}$ & $4.69 \pm 0.30$ & 7.2 & 53699.7766 & & $2 / 3$ & \\
\hline \multirow[t]{2}{*}{19} & 052.00069 & 07:39:09 & $+20: 48: 41$ & 10.43 & 10.85 & $21.288 \pm 7.664 \mathrm{e}^{-4}$ & $7.51 \pm 0.28$ & 11.6 & 53682.7645 & & $3 / 3$ & \\
\hline & & & & & & $29.627 \pm 1.666 \mathrm{e}^{-3}$ & $3.41 \pm 0.28$ & 5.2 & 53682.7835 & & & \\
\hline \multirow[t]{3}{*}{20} & 052.00159 & $07: 39: 20$ & $+21: 11: 22$ & 11.25 & 11.59 & $18.793 \pm 2.293 \mathrm{e}^{-3}$ & $9.59 \pm 0.42$ & 18.8 & 53699.7682 & & $2 / 3$ & \\
\hline & & & & & & $27.366 \pm 3.345 \mathrm{e}^{-3}$ & $6.69 \pm 0.42$ & 13.4 & 53699.7680 & & & \\
\hline & & & & & & $41.107 \pm 6.306 \mathrm{e}^{-3}$ & $3.19 \pm 0.32$ & 6.2 & 53699.7687 & & & \\
\hline 21 & 054.00014 & $07: 56: 31$ & $+21: 52: 29$ & 9.50 & 9.74 & $20.310 \pm 6.086 \mathrm{e}^{-5}$ & $2.89 \pm 0.14$ & 8.5 & 53702.8142 & A5 & $9 / 19$ & NSV 3816 \\
\hline & & & & & & $45.725 \pm 7.022 \mathrm{e}^{-5}$ & $2.16 \pm 0.14$ & 6.3 & 53702.8211 & & & \\
\hline & & & & & & $23.012 \pm 7.570 \mathrm{e}^{-5}$ & $2.32 \pm 0.14$ & 6.8 & 53702.8130 & & & \\
\hline 22 & 054.00075 & $07: 58: 27$ & $+21: 36: 24$ & 10.80 & 11.11 & $22.591 \pm 3.776 \mathrm{e}^{-5}$ & $7.21 \pm 0.026$ & 12.9 & 53702.8148 & & $5 / 19$ & \\
\hline 23 & 064.00006 & $08: 43: 26$ & $+16: 53: 00$ & 8.80 & 9.11 & $19.763 \pm 3.791 \mathrm{e}^{-1}$ & $6.84 \pm 0.34$ & 8.3 & 53812.5164 & & $1 / 2$ & \\
\hline 24 & 064.00050 & $08: 43: 45$ & $+17: 25: 00$ & 10.60 & 10.94 & $20.148 \pm 4.392 \mathrm{e}^{-1}$ & $7.47 \pm 0.44$ & 8.6 & 53812.5238 & & $1 / 2$ & \\
\hline 25 & 066.00003 & 08:44:07 & $+15: 55: 51$ & 8.56 & 8.71 & $54.890 \pm 9.400 \mathrm{e}^{-1}$ & $6.37 \pm 0.38$ & 10.6 & 53774.6133 & A0 & $1 / 2$ & \\
\hline 26 & 062.00060 & 09:05:56 & $+17: 47: 27$ & 10.90 & 11.50 & $38.163 \pm 8.580 \mathrm{e}^{-4}$ & $11.24 \pm 0.70$ & 7.4 & 53753.6878 & A5 & $3 / 9$ & \\
\hline & & & & & & $46.333 \pm 1.213 \mathrm{e}^{-3}$ & $7.94 \pm 0.68$ & 5.2 & 53753.7126 & & & \\
\hline 27 & 062.00030 & 09:09:53 & $+17: 41: 08$ & 10.18 & 10.40 & $21.098 \pm 3.614 \mathrm{e}^{-5}$ & $7.64 \pm 0.22$ & 9.0 & 53753.6725 & & $4 / 9$ & \\
\hline 28 & 107.00023 & $13: 10: 39$ & $-06: 25: 01$ & 10.25 & 10.51 & $20.214 \pm 1.921 \mathrm{e}^{-4}$ & $10.58 \pm 0.30$ & 10.4 & 53767.8800 & A0 & $4 / 10$ & \\
\hline 29 & 099.00087 & $15: 58: 40$ & $-19: 27: 14$ & 11.62 & 12.46 & $19.673 \pm 3.479 \mathrm{e}^{-1}$ & $19.86 \pm 0.92$ & 12.4 & 53903.6396 & & $1 / 1$ & \\
\hline 30 & 148.00060 & $16: 51: 54$ & $+07: 36: 44$ & 10.80 & 11.10 & $24.652 \pm 4.632 \mathrm{e}^{-4}$ & $27.71 \pm 0.64$ & 14.3 & 53812.7968 & A5 & $2 / 12$ & \\
\hline 31 & 012.00024 & $19: 51: 31$ & $-22: 23: 03$ & 9.84 & 10.20 & $27.102 \pm 7.279 \mathrm{e}^{-1}$ & $8.22 \pm 0.78$ & 6.0 & 53959.6073 & A6III & $1 / 4$ & \\
\hline 32 & 153.00128 & $20: 04: 47$ & $-20: 32: 05$ & 11.63 & 12.34 & $19.313 \pm 5.077 \mathrm{e}^{-1}$ & $43.72 \pm 2.92$ & 6.4 & 53919.7373 & & $1 / 1$ & \\
\hline 33 & 121.00214 & $21: 01: 43$ & $+16: 39: 37$ & 11.20 & 11.57 & $23.471 \pm 6.650 \mathrm{e}^{-4}$ & $9.20 \pm 0.48$ & 6.2 & 53559.7694 & & $2 / 10$ & \\
\hline 34 & 121.00043 & $21: 03: 25$ & $+15: 21: 26$ & 9.13 & 17.53 & $30.376 \pm 1.866 \mathrm{e}^{-3}$ & $3.61 \pm 0.16$ & 12.8 & 53750.7027 & & $3 / 10$ & \\
\hline 35 & 028.00439 & 21:54:03 & $+25: 11: 07$ & 12.85 & 13.54 & $19.445 \pm 6.747^{-5}$ & $32.02 \pm 1.16$ & 18.4 & 53575.7487 & & $5 / 9$ & \\
\hline & & & & & & $21.088 \pm 1.405 \mathrm{e}^{-4}$ & $15.39 \pm 1.02$ & 8.8 & 53575.7599 & & & \\
\hline & & & & & & $8.847 \pm 1.238 \mathrm{e}^{-3}$ & $9.79 \pm 1.02$ & 5.7 & 53575.7475 & & & \\
\hline 36 & 028.01026 & 22:01:08 & $+24: 44: 33$ & 13.01 & 13.42 & $33.008 \pm 5.843 \mathrm{e}^{-5}$ & $36.45 \pm 1.72$ & 11.5 & 53575.7736 & & $5 / 9$ & \\
\hline 37 & 003.00147 & $22: 01: 53$ & $-12: 28: 52$ & 12.24 & 12.23 & $17.731 \pm 2.283 \mathrm{e}^{-4}$ & $32.24 \pm 1.14$ & 18.5 & 53947.6845 & & $4 / 4$ & \\
\hline & & & & & & $41.446 \pm 1.392 \mathrm{e}^{-3}$ & $12.43 \pm 1.28$ & 7.4 & 53947.7181 & & & \\
\hline & & & & & & $30.608 \pm 8.642 \mathrm{e}^{-4}$ & $9.26 \pm 1.26$ & 5.4 & 53947.7213 & & & \\
\hline 38 & 138.00022 & $22: 05: 55$ & $+28: 02: 32$ & 9.54 & 10.05 & $17.767 \pm 6.301 \mathrm{e}^{-5}$ & $5.11 \pm 0.20$ & 12.0 & 53781.7689 & A3 & $6 / 13$ & \\
\hline & & & & & & $18.090 \pm 9.286 \mathrm{e}^{-5}$ & $3.50 \pm 0.20$ & 8.2 & 53781.7733 & & & \\
\hline 39 & 138.00110 & $22: 09: 41$ & $+28: 12: 40$ & 10.80 & 11.30 & $16.877 \pm 1.751 \mathrm{e}^{-4}$ & $7.87 \pm 0.32$ & 12.6 & 53584.7897 & & $4 / 13$ & \\
\hline 40 & 030.00019 & $22: 59: 23$ & $+37: 14: 33$ & 9.26 & 9.65 & $20.199 \pm 9.589 \mathrm{e}^{-4}$ & $5.21 \pm 0.20$ & 13.5 & 53657.6049 & $\mathrm{~F} 2$ & $4 / 8$ & \\
\hline & & & & & & $29.390 \pm 1.564 \mathrm{e}^{-3}$ & $2.60 \pm 0.18$ & 6.7 & 53657.6029 & & & \\
\hline & & & & & & $19.757 \pm 1.579 \mathrm{e}^{-3}$ & $2.86 \pm 0.20$ & 7.4 & 53657.5702 & & & \\
\hline 41 & 030.00195 & 23:02:06 & $+36: 30: 28$ & 11.51 & 11.91 & $22.278 \pm 5.635 \mathrm{e}^{-4}$ & $13.56 \pm 0.42$ & 21.4 & 53657.5695 & & $5 / 8$ & \\
\hline & & & & & & $18.802 \pm 1.529 \mathrm{e}^{-3}$ & $5.14 \pm 0.40$ & 8.1 & 53657.6120 & & & \\
\hline
\end{tabular}

Notes.

${ }^{\text {a }}$ Combination of TAOS field ID and TAOS star ID.

${ }^{\mathrm{b}}$ We doubled amplitudes derived by PERIOD04.

${ }^{c} \mathrm{~S} / \mathrm{N}$ of frequencies derived using PERIOD04.

d The number of identifications/the number of zipper runs. Note that we did not count zipper runs observed by only one telescope.

e The B8 star found to be an A5 star as explained in the text.

${ }^{\mathrm{f}}$ The G5 star found to be an F0 star as explained in the text. 


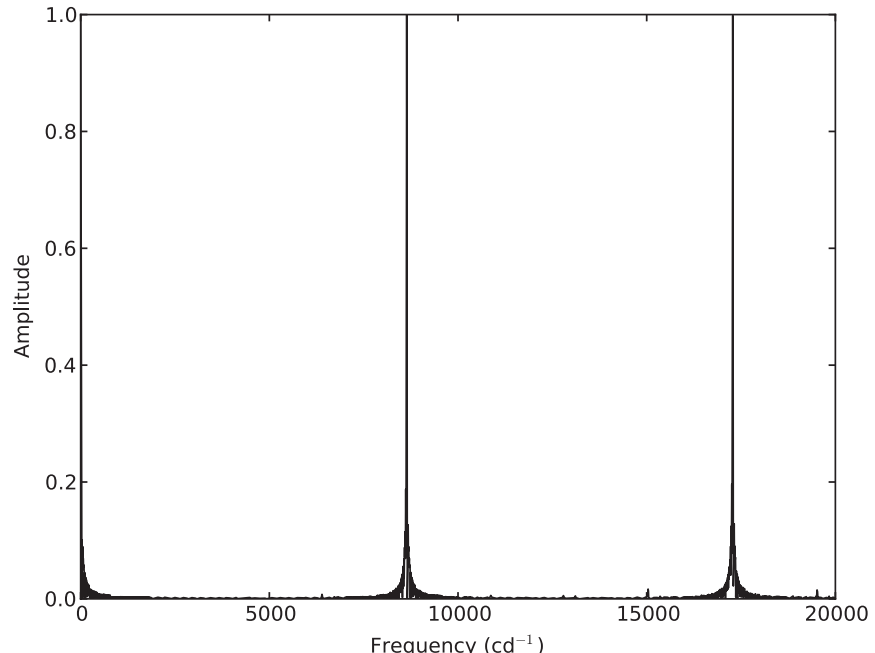

Figure 3. Example of a spectral window of a single zipper run. Peaks with regular intervals (two times of the Nyquist frequency) appear because points are equally spaced in a single zipper run.

find $m_{V}, m_{B}$, and spectral types, we used Centre de Données astronomiques de Strasbourg (CDS) Web service (Genova et al. 2000).

In Table 3, we also provide the number of the total observations (i.e., the number of the total zipper runs) and the number of identifications by the FFT analysis for each TAOS $\delta$ Sct star. Since the TAOS telescopes observe same fields multiple times, we have multiple light curves for stars in the fields. Note that we applied the FFT algorithm to each light curve to detect periodic signals. Nevertheless, as the table shows, not every light curve of the TAOS $\delta$ Sct stars was confirmed to have periodic signals. This is mainly because of the poor quality of some of the light curves caused by trends and noise (i.e., unstable weather, telescope vibration, etc.). Although we removed most of the trends using PDT, it is nearly impossible to recover the intrinsic periodic signal of a few millimagnitudes in the presence of large systematic errors.

\subsection{Spectral Windows and Power Spectra of TAOS $\delta$ Sct Stars}

In Figure 3, we show an example of a spectral window of a single zipper run. Since the observational times are almost equally spaced, peaks with regular intervals (two times the Nyquist frequency) are present in the spectral window (Deeming 1975). The Nyquist frequency is $4320 \mathrm{~Hz}$ since the gap between each consecutive binned data is $10 \mathrm{~s}$.

Figures 4-6 show the spectral windows along with the power spectra of three TAOS $\delta$ Sct stars. Stars included in these figures are 020.00141 (Figure 4), 121.00043 (Figure 5), and 054.00014 (Figure 6), respectively. The top panels in each figure show the spectral windows, and the bottom panels (and the middle panel in Figure 6) show the power spectra of the stars. Dashed lines indicate the detected frequencies. Note that we improved the detected frequencies by fitting a combination of sine waves using PERIOD04. Thus the improved frequencies could be slightly shifted from the original peaks in the power spectra (e.g., see the bottom left panel in Figure 4) after the fitting.

As Table 3 shows, the star with ID 020.00141 was identified only once and is relatively fainter $\left(m_{V}=11.40\right)$ than other TAOS $\delta$ Sct stars. Its amplitude is one of the smallest amplitudes and its detected frequency $\mathrm{S} / \mathrm{N}$ is the lowest among TAOS $\delta$ Sct stars. Thus the power spectrum of this star represents one of
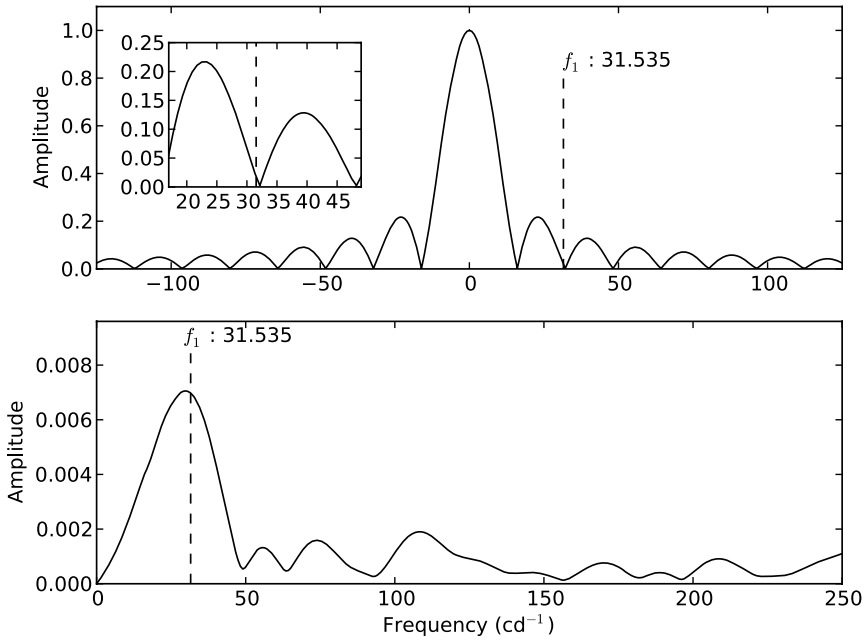

Figure 4. Spectral window and the power spectrum of the star ID 020.00141. The top panel shows the spectral window, and the bottom panel shows the power spectrum of the star. The dashed line shows the detected frequency. In the top panel, we magnified the spectral window to clearly show the detected frequency.
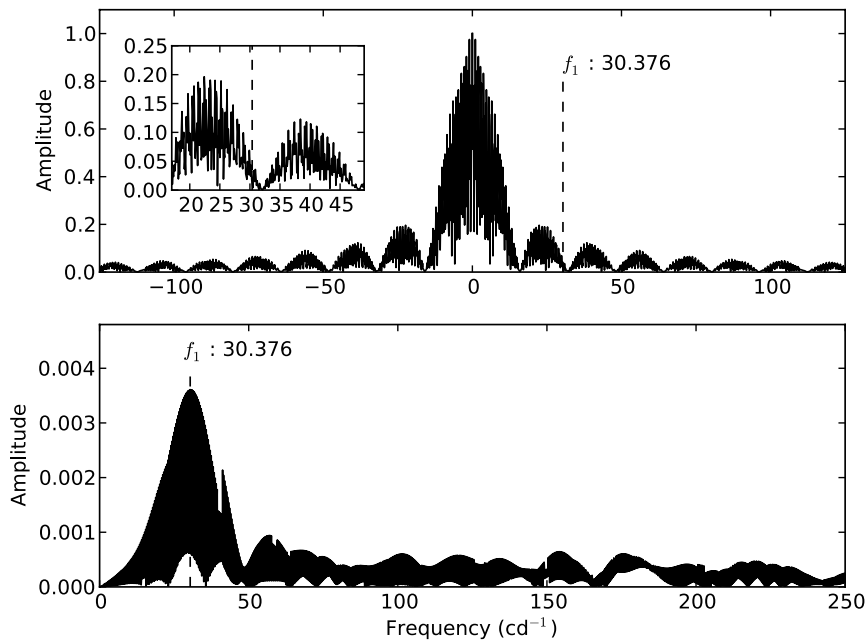

Figure 5. Spectral window and the power spectrum of the star ID 121.00043. The top panel shows the spectral window, and the bottom panel shows the power spectrum of the star. The dashed line shows the detected frequency. In the top panel, we magnified the spectral window to clearly show the detected frequency.

the "worst-case scenarios." We detected one single frequency for this star. The star with ID 121.00043 was identified three times and is relatively bright $\left(m_{V}=9.13\right)$. The spectral window and the power spectrum of the star could represent a "moderatelevel scenario." We detected one frequency for this star. Finally, the star with ID 054.00014 was identified nine times and is relatively bright $\left(m_{V}=9.50\right)$. Thus its spectral window and power spectrum represent one of the "best-case scenarios." Using PERIOD04, we detected three frequencies for this star. As can be seen from the figures, there are no significant peaks in the spectral windows at the detected frequencies.

\subsection{Spectroscopy of Two Peculiar Spectral Type $\delta$ Sct Stars}

As we mentioned in the previous section, we found two peculiar spectral-type $\delta$ Sct stars which have B8 and G5 spectral types. These are the bluest and reddest spectral types of $\delta$ Sct stars ever detected. The spectral type of the B8 star was extracted from the Henry Draper Catalogue and Extension (HD; Cannon \& Pickering 1993). Unfortunately, we could not find any spectroscopic literature for the G5 star so we suspect that the 


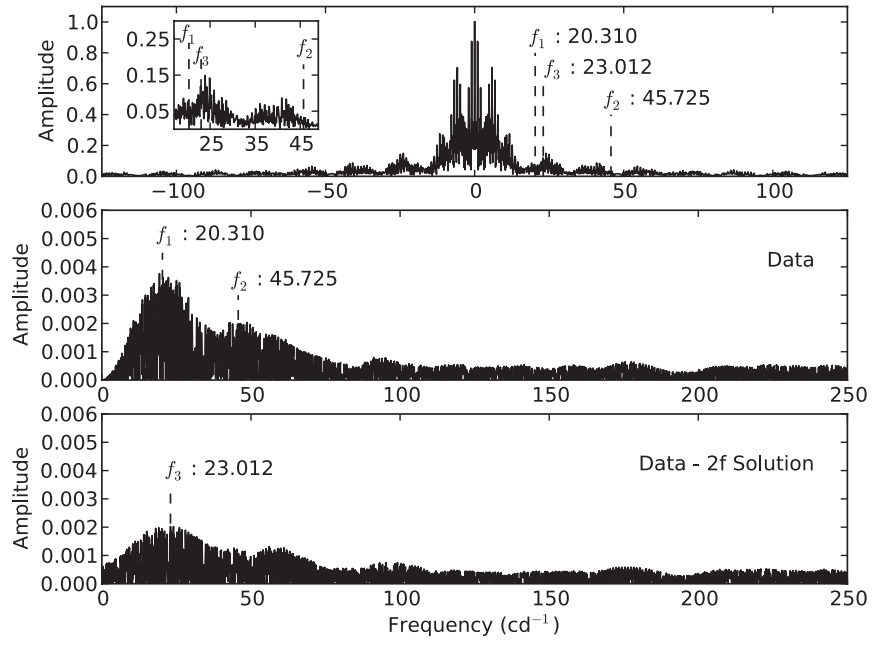

Figure 6. Spectral window and the power spectrum of the star ID 054.00014. We detected three frequencies (dashed lines) using PERIOD04. The top panel shows the spectral window. The middle panel shows the first two frequencies. The bottom panel is the power spectrum after whitening the two frequencies. In the top panel, we magnified the spectral window to clearly show the detected frequencies.

spectral type is most likely derived from its color information. The spectral type of the G5 star was extracted from SIMBAD. To confirm their spectral types, we observed the two stars with spectroscopic instruments.

For the $\mathrm{B} 8$ star, we used the $\mathrm{BOES}^{20}$ of the $1.8 \mathrm{~m}$ telescope at the Bohyunsan Optical Astronomy Observatory (BOAO), South Korea (Kim et al. 2007). We used IRAF (Tody 1986, 1993) for the reduction of the obtained spectroscopic data. Figure 7 shows the normalized spectrum of the B8 candidate star. We indicate several important spectral lines in the figure. As the figure shows, the $\mathrm{Ca}$ II $\mathrm{K}$ line is very strong which is typical for A-type stars (Gray \& Garrison 1987). B-type stars do not show such a strong

\footnotetext{
${ }^{20}$ BoaO Echelle Spectrograph.
}

Ca II $\mathrm{K}$ line. The spectrum also shows weak metallic lines (e.g., $\mathrm{Ca}$ I and $\mathrm{Mg}$ II lines) which are usually presented in A-type stars. Based on the strength of the Ca II $\mathrm{K}$ line, hydrogen lines, and metallic lines, the star is likely an A5-type star although the classification of sub-class is rather uncertain due to the low $\mathrm{S} / \mathrm{N}$ of the spectrum.

In addition, to observe the G5 star, we used the FAST instrument mounted at the Fred Lawrence Whipple Observatory (FLWO) $1.5 \mathrm{~m}$ telescope, Mount Hopkins in Arizona (Fabricant et al. 1998). After comparing the observed data with standard spectral libraries (Pickles 1998), we found that the spectral type of the star is not a G5 but an F0. Therefore, the star is likely a typical $\delta$ Sct star.

\section{SUMMARY}

We analyzed the TAOS two-year data accumulated during 2005 and 2006 observations in order to find short-period variables. Using the TAOS photometry pipeline, we created photometric light curves. We removed systematic trends commonly appeared in the light curves using PDT. To detect periodic signals in the detrended light curves, we applied the FFT to each light curve. FFT is a simple but powerful algorithm for detection of periodic signals when data points are evenly spaced. We then chose light curves which possess a frequency (or frequencies) whose power is five times larger than the standard deviation of powers of all background frequencies in the power spectrum derived using FFT. We visually checked the light curves and raw images of all candidates to remove false positives caused by moving asteroids, photometry defects, etc. We also removed candidates which were detected by only one of the three telescopes. All remaining 41 variable candidates have periods about an hour and amplitudes less than a few hundredth of a magnitude, which strongly suggests that they are LADS.

We cross-matched the detected $\delta$ Sct candidate stars with many astronomical catalogs to extract additional information (e.g., magnitude, spectral type, variability type, etc.). As a result, we found that 14 stars have spectral types from A to F, which

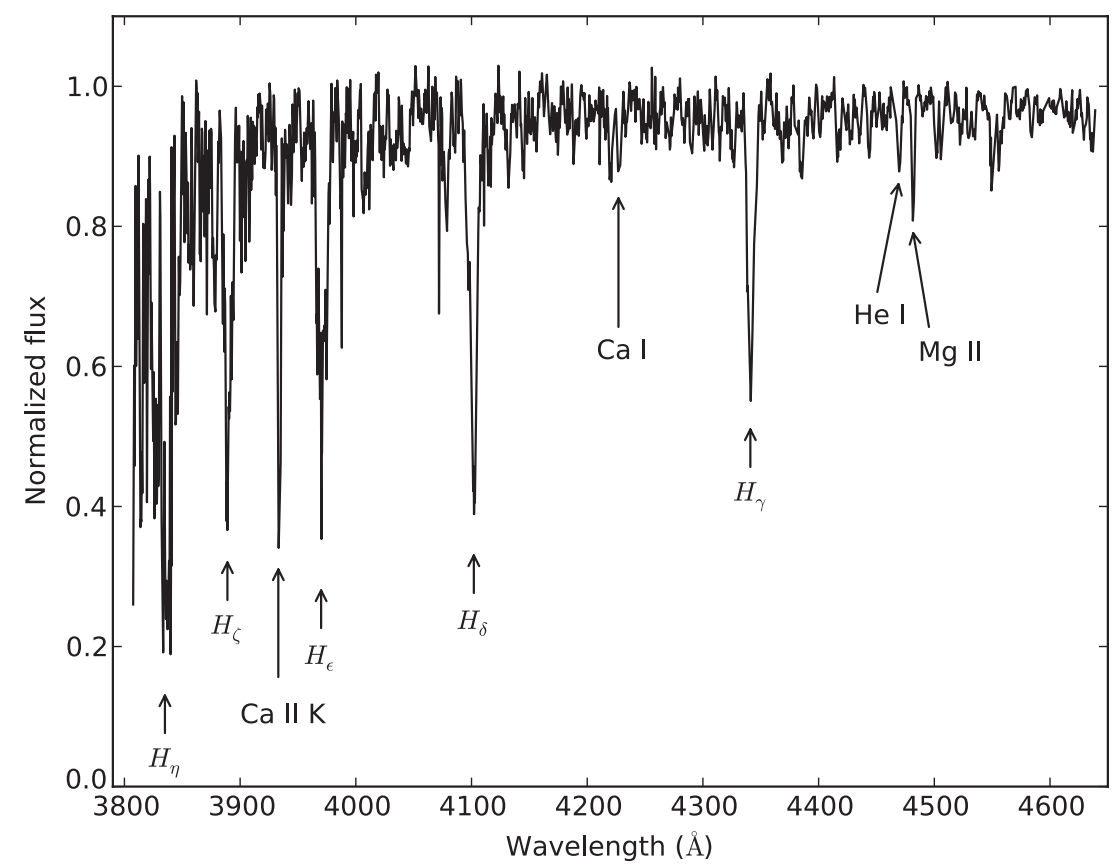

Figure 7. Normalized spectrum of the B8 candidate star. There are strong Ca II $\mathrm{K}$ line and weak metallic lines, which is typical for A-type stars. The star is likely an A5-type star rather than a B8-type star. 
are typical spectral types for $\delta$ Sct stars. The rest of the detected $\delta$ Sct stars do not have spectral information.

The light curves of TAOS $\delta$ Sct stars are accessible at the Time Series Center (TSC, http://timemachine.iic.harvard.edu), Initiative in Innovative Computing (IIC) at Harvard. PERIOD04 project files of each star is also provided. The project files contain complete light-curve data, power spectrum, frequency, and amplitude information.

Y.-I. Byun acknowledges the support of the National Research Foundation of Korea through Grant 2009-0075376. The work at National Central University was supported by the grant NSC 96-2112-M-008-024-MY3. Work at Academia Sinica was supported in part by the thematic research program AS-88-TPA02. Work at the Harvard College Observatory was supported in part by the National Science Foundation under grant AST0501681 and by NASA under grant NNG04G113G. S.L.M.'s work was performed under the auspices of the U.S. Department of Energy by Lawrence Livermore National Laboratory in part under Contract W-7405-Eng-48 and by Stanford Linear Accelerator Center under Contract DE-AC02-76SF00515. K. H. Cook's work was performed under the auspices of the U.S. Department of Energy by Lawrence Livermore National Laboratory in part under Contract W-7405-Eng-48 and in part under Contract DE-AC52-07NA27344. We also thank J. D. Hartman at Harvard-Smithsonian Center for Astrophysics for useful discussion.

The detrending and the analysis of data sets in this paper were run on the Odyssey cluster supported by the FAS Research Computing Group at the Harvard. This research has made use of the SIMBAD database, operated at CDS, Strasbourg, France. IRAF is distributed by the National Optical Astronomy Observatories, which are operated by the Association of Universities for Research in Astronomy, Inc., under cooperative agreement with the National Science Foundation.

\section{REFERENCES}

Alcock, C., et al. 2003, Earth Moon Planets, 92, 459

Arentoft, T., et al. 2001, A\&A, 374, 1056

Balona, L. A. 2004, in IAU Symp. 224, The A-Star Puzzle, Pulsations of A stars, ed. J. Zverko, J. Ziznovsky, S. J. Adelman, \& W. W. Weiss (Dordrecht: Kluwer), 325

Balona, L. A., \& Evers, E. A. 1999, MNRAS, 302, 349

Breger, M. 2000a, in ASP Conf. Ser. 210, Delta Scuti and Related Stars, $\delta$ Scuti Stars (Review), ed. M. Breger \& M. Montgomery (San Francisco, CA: ASP), 3

Breger, M. 2000b, MNRAS, 313, 129

Breger, M., \& Pamyatnykh, A. A. 1998, A\&A, 332, 958

Breger, M., et al. 1993, A\&A, 271, 482

Breger, M., et al. 2002, MNRAS, 336, 249

Breger, M., et al. 2005, A\&A, 435, 955

Brigham, E. O. 1974, The fast Fourier Transform (Englewood Cliffs, NJ: Prentice-Hall)

Brown, T. M., \& Gilliland, R. L. 1994, ARA\&A, 32, 37
Bruntt, H., et al. 2007, A\&A, 461, 619

Bush, T. C., \& Hintz, E. G. 2008, AJ, 136, 1061

Buzasi, D. L., et al. 2005, ApJ, 619, 1072

Cannon, A. J., \& Pickering, E. C. 1993, VizieR Online Data Catalog, 3135,0

Chapellier, E., et al. 2004, A\&A, 426, 247

Chen, W. P., et al. 2007, in IAU Symp. 236, Search for Small Trans-Neptunian Objects by the TAOS Project, ed. G. B. Valsecchi, D. Vokrouhlický, \& A Milani (Dordrecht: Kluwer), 65

Christiansen, J. L., et al. 2007, MNRAS, 382, 239

de Boor, C. 1978, A Practical Guide to Splines (New York: Springer), 87

Deeming, T. J. 1975, Ap\&SS, 36, 137

Fabricant, D., Cheimets, P., Caldwell, N., \& Geary, J. 1998, PASP, 110, 79

Garrido, R., Garcia-Lobo, E., \& Rodriguez, E. 1990, A\&A, 234, 262

Genova, F., et al. 2000, A\&AS, 143, 1

Glebocki, R., \& Stawikowski, A. 2000, Acta Astron., 50, 509

Gray, R. O., \& Garrison, R. F. 1987, ApJS, 65, 581

Handler, G., Gray, R. O., \& Shobbrook, R. R. 2000, IBVS, 4876, 1

Hartman, J. D., et al. 2008, ApJ, 675, 1254

Henry, G. W., \& Fekel, F. C. 2005, AJ, 129, 2026

Henry, G. W., et al. 2001, AJ, 122, 3383

Høg, E., et al. 2000, A\&A, 355, L27

Jain, A. K., Murty, M. N., \& Flynn, P. J. 1999, ACM Comput. Surv., 31, 264

Jin, H., et al. 2003, A\&A, 404, 621

Kang, Y. B., et al. 2007, PASP, 119, 239

Kharchenko, N. V. 2001, Kinematika Fiz. Nebesnykh Tel, 17, 409

Kim, D.-W., et al. 2009, MNRAS, 397, 558

Kim, K.-M., et al. 2007, PASP, 119, 1052

Lasker, B. M., et al. 2008, AJ, 136, 735

Lehner, M. J., et al. 2009, PASP, 121, 138

McNamara, D. H., Clementini, G., \& Marconi, M. 2007, AJ, 133, 2752

Monet, D. G., et al. 2003, AJ, 125, 984

Moya, A., Garrido, R., \& Dupret, M. A. 2004, A\&A, 414, 1081

Paunzen, E. 2004, in IAU Symp. 224, The A-Star Puzzle, The $\lambda$ Bootis stars, ed. J. Zverko, J. Ziznovsky, S. J. Adelman, \& W. W. Weiss (Dordrecht: Kluwer), 443

Perryman, M. A. C., \& ESA (ed.) 1997, The HIPPARCOS and TYCHO Catalogues. Astrometric and Photometric Star Catalogues Derived from the ESA HIPPARCOS Space Astrometry Mission (ESA Special Publication, Vol. 1200; Noordwijk: ESA)

Pickles, A. J. 1998, PASP, 110, 863

Pigulski, A., et al. 2009, Acta Astron., 59, 33

Pojmanski, G., et al. 2006, VizieR Online Data Catalog, 2264, 0

Pribulla, T., et al. 2008, MNRAS, 391, 343

Ripepi, V., et al. 2003, A\&A, 408, 1047

Rodríguez, E., \& López-González, M. J. 2000, A\&A, 359, 597

Rodríguez, E., López-González, M. J., \& López de Coca, P. 2000, A\&AS, 144, 469

Rodríguez, E., et al. 1996, A\&A, 307, 539

Samus, N. N., et al. 2009, VizieR Online Data Catalog, 1, 2025

SAO Staff 1995, VizieR Online Data Catalog, 1131, 0

Skiff, B. A. 2009, VizieR Online Data Catalog, 1, 2023

Tody, D. 1986, Proc. SPIE, 627, 733

Tody, D. 1993, in ASP Conf. Ser. 52, Astronomical Data Analysis Software and Systems II, IRAF in the Nineties, ed. R. J. Hanisch, R. J. V. Brissenden, \& J. Barnes (San Francisco, CA: ASP), 173

Wenger, M., et al. 2000, A\&AS, 143, 9

Zhang, Z. W., et al. 2009, PASP, 121, 1429

Zima, W., et al. 2002, in ASP Conf. Ser. 259, Radial and Nonradial Pulsationsn as Probes of Stellar Physics, The Delta Scuti Network: Steps Towards Successful Asteroseismology of Delta Scuti Stars, ed. C. Aerts, T. R. Bedding, \& J. Christensen-Dalsgaard (San Francisco, CA: ASP), 598 